\title{
Construction of 2D lateral pseudoheterostructures by strain engineering
}

Haifeng Feng ${ }^{1,2,8}$, Jincheng Zhuang ${ }^{1,8}$, Ashley D Slattery ${ }^{3}$, Liang Wang ${ }^{1}$, Zhongfei $\mathrm{Xu}^{2}$, Xun $\mathrm{Xu}^{1}$, David Mitchell ${ }^{4}$, Tian Zheng ${ }^{5}$, Songlin $\mathrm{Li}^{6}$, Michael Higgins ${ }^{5}$, Long Ren ${ }^{1}$, Ziqi Sun ${ }^{7}$, Shi Xue $\mathrm{Dou}^{1}$, Yi Du ${ }^{1,2}$, and Weichang $\mathrm{Hao}^{2}$

${ }^{1}$ Institute for Superconducting and Electronic Materials, and UOW-BUAA Joint Research Centre, University of Wollongong, Wollongong, NSW 2500, Australia

${ }^{2}$ Department of Physics and Key Laboratory of Micro-Nano Measurement, Manipulation and Physics, Ministry of Education, and UOW-BUAA Joint Research Centre, Beihang University, Beijing 100191, China

${ }^{3}$ Flinders Centre for NanoScale Science and Technology, School of Chemical and Physical Sciences, Flinders University, Bedford Park, South Australia 5042, Australia

${ }^{4}$ Electron Microscopy Centre, University of Wollongong, Wollongong, NSW 2500, Australia

${ }^{5}$ Intelligent Polymer Research Institute and ARC Centre of Excellence for Electromaterials Science, University of Wollongong, Wollongong, NSW 2500, Australia

${ }^{6}$ School of Electronic Science and Engineering, Nanjing University, Nanjing, Jiangsu 210023, China

${ }^{7}$ School of Chemistry, Physics and Mechanical Engineering, Queensland University of Technology, Brisbane, Queensland 4000, Australia

${ }^{8}$ These authors contributed equally to this work.

E-mail: ydu@uow.edu.au,whao@buaa.edu.cn

Keywords: pseudoheterostructure, lateral, 2D materials, $\mathrm{BiOBr}$, strain, photodetector 
Abstract. Two-dimensional (2D) lateral heterostructures host unconventional physical properties due to their controllable band-offset tuning and interfacial sensitive characteristic. The lattice mismatch results in the difficulties to construct the perfect atomic interface in such 2D lateral heterostructures, which in turn limits their desirable properties and performances in applications. In this work, strain-modulated 2D lateral pseudoheterogeneous structures are designed and realized in the single-component $2 \mathrm{D} \mathrm{BiOBr}$ nanosheets by taking advantage of their strain-sensitive crystal and electronic structures. The pseudoheterogeneous interface without atomic mismatch can be feasibly modulated by local strain distribution, which exhibits similar local electronic band structure of corresponding heterostructures. Significant enhancement in charge separation at the pseudoheterostructure was demonstrated under visible light irradiation, which is given rise to the controllable electronic band alignment across the interface. The construction of the lateral pseudoheterostructure offers a feasible and promising way to build unprecedented two-dimensional systems with exciting properties.

\section{Introduction}

Discontinuities at interfaces in heterogeneous structures can lead to exciting and possibly non-trivial properties, due to broken symmetries at the interfaces. Through constructing heterostructures, one can feasibly engineer and manipulate electronic, optical, and magnetic phases at so-called heterointerfaces, and thus, generate unusual properties and new phenomena [1,2]. Numerous breakthroughs have been achieved by taking advantage of vertical heterostructures in previous studies. For examples, naturally formed heterostructures give birth to high-temperature superconductivity $\left(\right.$ High- $\left.T_{\mathrm{C}}\right)$ and topological states in 
copper-oxide-based superconductors and topological insulators, respectively [3,4]. Artificial heterostructures were also constructed in laminated $\mathrm{LaAlO}_{3} / \mathrm{SrTiO}_{3}$ films, in which magnetic order and superconductivity surprisingly coexist $[5,6]$. Very recently, fabrication of vertical van de Waals (vdW) heterogeneous structures were successfully achieved by using atomically thin layer materials, including graphene and $h$ - $\mathrm{BN}$, which drew immediate attention [7-11]. Besides vertical heterostructures, lateral heterostructures with two materials joined laterally have also drawn great attention [12]. The research in this field was boosted soon after single-layer-thick 2D lateral heterostructures were successfully fabricated, as they possess controllable band-offset tuning and can be used in electronics, optoelectronics, and catalysis. Lateral interfaces in 2D lateral heterostructures are constructed from covalent bonds and not linked by vdW forces. Less electron and phonon scattering centers are expected across the interface. Electron hopping and band alignment in such lateral heterostructures are therefore less affected by the interfaces, which, in turn, promotes charge carrier transport across the lateral interfacial junctions. These advantages of 2D lateral heterostructures have been observed and verified in graphene/ $h$-BN lateral heterogeneous structures. In these systems, high field-effect mobility of charge carriers and low hysteresis behavior have been demonstrated [13,14]. 2D lateral heterostructures based on two different transition-metal dichalcogenides (TMD) were also successfully fabricated, exhibiting lateral $p$ - $n$ diodes behaviors or staggered band alignment. A broad range of applications based on 2D lateral TMD heterostructures, therefore, have been proposed, such as logic circuits, field-effect transistors, and photodetectors [15-18].

Despite their appealing advantages, great challenges remain in constructing 2D lateral 
heterostructures with the desired properties. A clean and atomically sharp interface is the essential requirement to achieve exotic characteristics. To achieve this goal, sophisticated and costly manufacturing methods such as molecular beam epitaxy, lithography, and chemical vapor deposition are necessary for fabricating these high-quality interfaces [12,19]. It is still extremely difficult to achieve 2D lateral heterostructures with a large area, instead, most epitaxially-grown samples possess small heterogeneous domains. The other limitation originates from lattice mismatch at the lateral heterointerface. The candidate materials for $2 \mathrm{D}$ lateral heterostructures must have similar crystal structures and lattice constants. This is the reason why very few 2D lateral heterostructures have been reported so far [20]. An alternative strategy to overcome these limitations is to develop a "lateral pseudoheterostructure" [21,22]. Generally, the pseudoheterostructures have a single chemical component but show spatial variation in their physical properties, which offers a promising way to build $2 \mathrm{D}$ systems that show the functionalities of $2 \mathrm{D}$ lateral heterostructures but overcome the problems of lattice mismatch.

Here, we report a 2D lateral pseudoheterostructure with an electronic heterogeneous interface constructed from single-component $\mathrm{BiOBr} 2 \mathrm{D}$ nanosheets by strain engineering. As a typical bismuth oxyhalide $(\mathrm{BiOX}$, where $\mathrm{X}=\mathrm{Cl}, \mathrm{Br}, \mathrm{I})$ [23-26], $\mathrm{BiOBr}$ has great potential for use in photoenergy conversion applications, owing to its indirect band gap of $2.8 \mathrm{eV}$ [27-30]. Taking advantage of their strain-sensitive electronic structure, few-layer-thick $\mathrm{BiOBr}$ nanosheets were successfully prepared with controlled spatial distributions of local electronic structures by manipulating the strain distribution. The position and characteristics of the electronic heterogeneous interface can also be tuned by adjusting the strain distribution 
in the nanosheets. Effective separation of charge carriers at the electronic heterointerface is then demonstrated in this lateral pseudoheterostructure, owing to the appropriate band alignment at the electronic heterogeneous interface. Its excellent photoresponse and enhanced photocurrent suggest that such lateral pseudoheterostructures are potential candidates for superior optoelectronic devices.

\section{Results and Discussion}
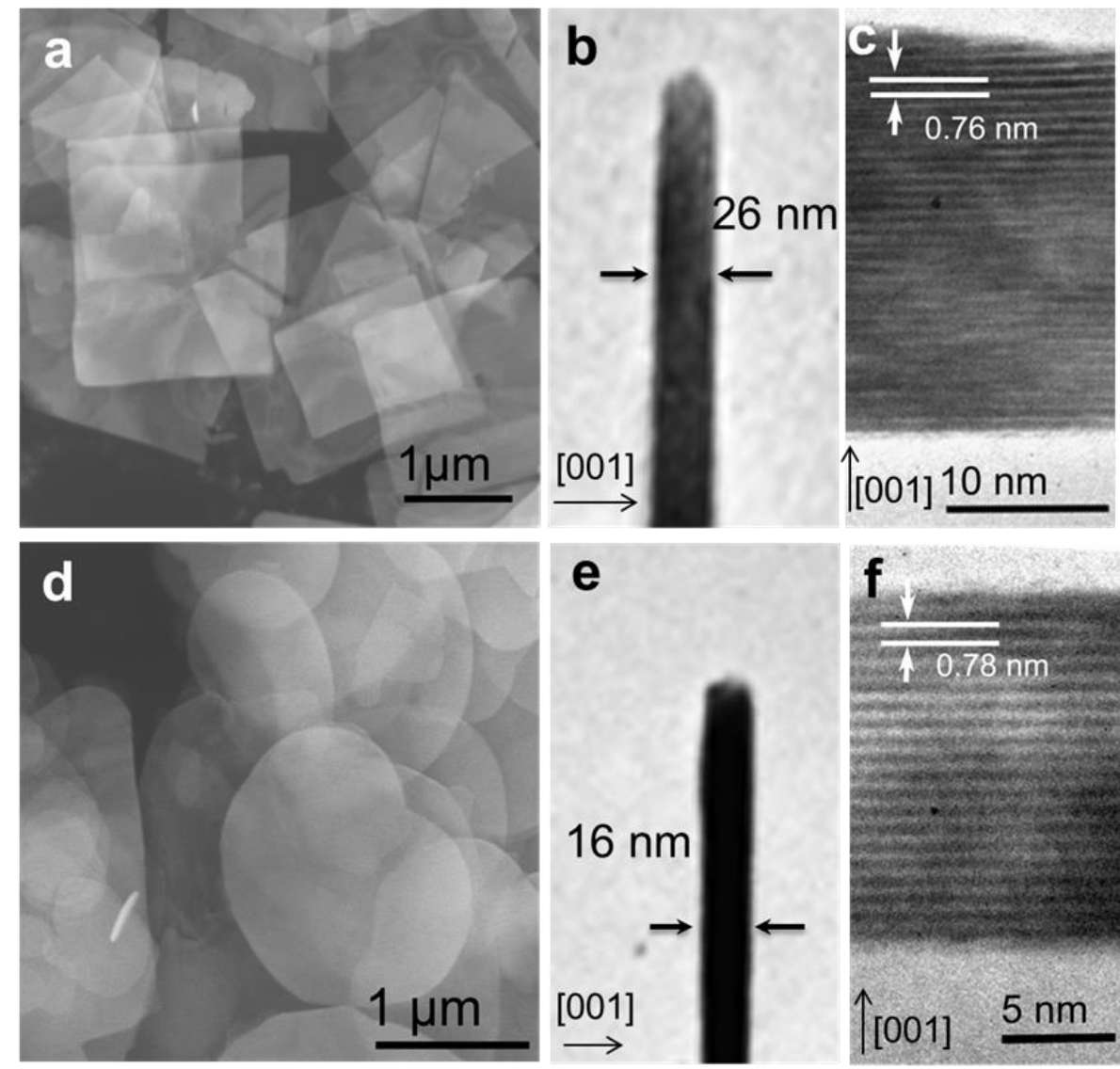

Figure 1. (a) SEM image of BiOBr-square nanosheets. (b) and (c) Cross-sectional TEM images of a single BiOBr-square nanosheet. (d) SEM image of BiOBr-circle nanosheets. (e) and (f) Cross-sectional TEM of a single BiOBr-circle nanosheet. 
The as-prepared $\mathrm{BiOBr} 2 \mathrm{D}$ nanosheets were synthesized by the hydrothermal method with cetyltrimethylammonium bromide $(\mathrm{CTAB})$ as the structural template at different $\mathrm{pH}$ values (with the $\mathrm{pH}$ value varying from 7 to 2). The morphologies of the $\mathrm{BiOBr}$ nanosheets synthesized at $\mathrm{pH}$ values of 7 and 2 are revealed by the scanning electron microscope (SEM) images (figure 1(a) and (d)). They prove that the $\mathrm{BiOBr}$ nanosheets are approximately several micrometers and undergo a shape transition from square $(\mathrm{pH}=7)$ to circle $(\mathrm{pH}=2)$ (see the transitional shapes in figure S1 in Supporting Information). The concentration of hydroxide is thus expected to be the key factor that determines the shape of the $2 \mathrm{D} \mathrm{BiOBr}$ nanosheets. Despite their different shapes, all the nanosheets display a layered structure, which is clearly confirmed in the cross-sectional transmission electron microscopy (TEM) images in figure 1. The thickness of the BiOBr-square and BiOBr-circle show similar thickness of 15-40 nm. The large area-to-thickness ratio reflects their 2D nature. The interlayer distances for the BiOBr-square and BiOBr-circle nanosheets are $\sim 0.76 \mathrm{~nm}$ and $\sim 0.78 \mathrm{~nm}$, respectively, which correspond to the spacing of $\mathrm{BiOBr}(001)$ face. This indicates that the $\mathrm{BiOBr}$ nanosheets have a preferred growth orientation normal along the [001] direction. Our X-ray diffraction (XRD), SEM, TEM, and energy dispersive spectroscopy (EDS) measurements verify that all BiOBr nanosheets are pure phase without any detectable impurities or structural defects (figures S3 and S4 in Supporting Information). According to the Wulff theorem [31], BiOBr crystals preferentially form in a tetragonal shape (square) to maximize the exposure of the (001) face; this minimizes the total surface energy, because the (001) face has the lowest surface energy of all the faces. In a neutral environment $(\mathrm{pH}=7), \mathrm{BiOBr}$ nanosheets take on a square shape to favor the lowest energy [32,33]. As the concentration of hydroxide decreased, however, 
the growth of $\{110\}$ facets were promoted in the $\mathrm{BiOBr}$ nanosheets, and consequently, led to their shape gradually changing from square to circle [34].
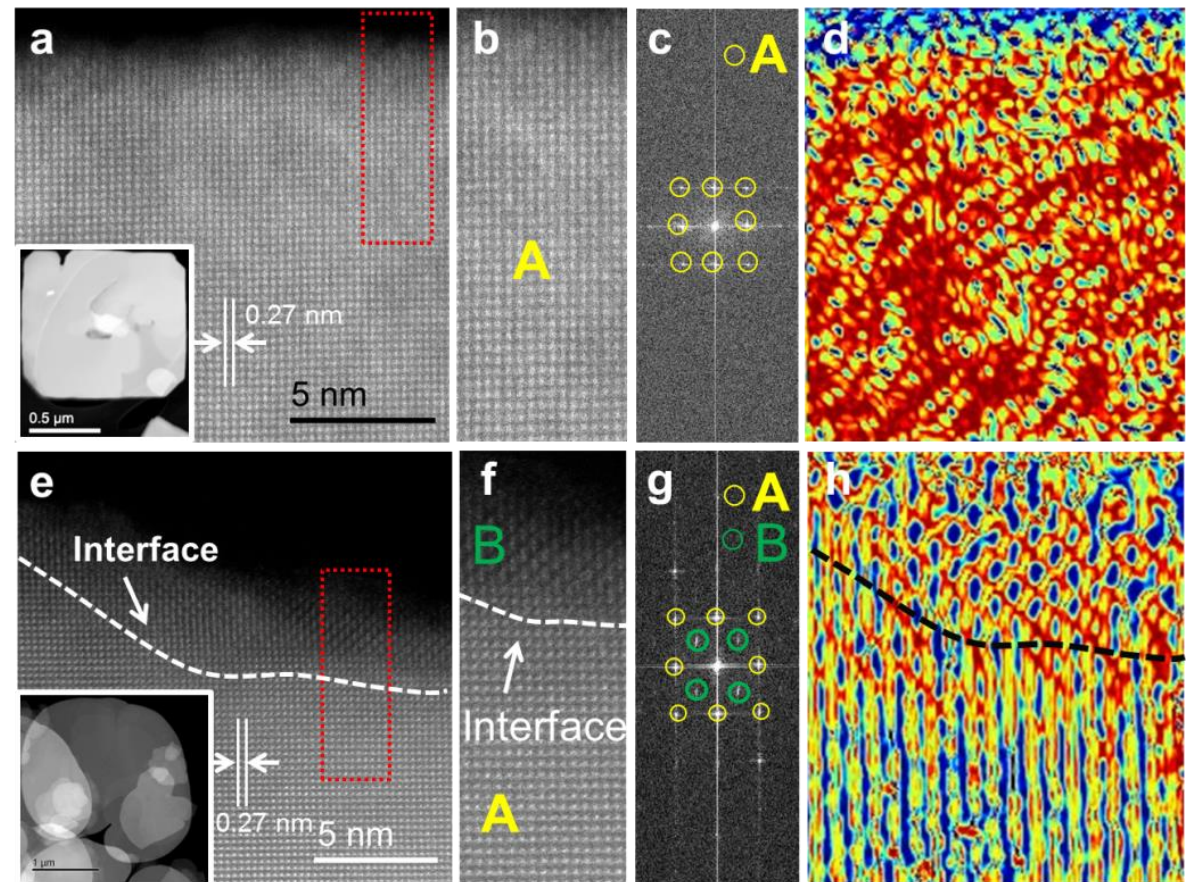

Figure 2. (a) $\mathrm{HAADF}$ image of $\mathrm{BiOBr}$-square, showing a homogeneous crystal structure (inset: lower magnification to show whole nanosheet). (b) Enlarged image from the selected area in a. (c) Corresponding FFT pattern of the area in b. (d) Strain mapping of BiOBr-square by GPA. (e) HAADF image of BiOBr-circle, showing an interface of the pseudoheterostructure (inset: lower magnification to show whole nanosheet). (f) Enlarged image from the selected area in e. (g) Corresponding FFT pattern of the area in f. (h) Strain mapping of BiOBr-square by GPA. In the image of GPA, the blue zones are under compressive strain, the red zones are under tensile strain, and the green zones are not strained. 
Figure 2 shows TEM images of $\mathrm{BiOBr}$ nanosheets with both square and circle shape that were collected in high-angle annular dark field (HAADF) mode. The atomic arrangements of the nanosheets are clearly revealed and demonstrate their 2D single-crystal nature. Interestingly, these $\mathrm{BiOBr}$ nanosheets have distinct local atomic features associated with their shapes, especially in the areas close to the edges of the nanosheets. A homogeneous atomic arrangement is observed across the whole $\mathrm{BiOBr}$-square nanosheet, including edge areas, as shown in figure 2(b). No obvious difference has been found in the atomic fringes across the whole square nanosheet. The corresponding fast Fourier transform (FFT) pattern is shown in figure 2(c) (pattern A across the whole nanosheet). Only one set of patterns can be observed. In contrast, the $\mathrm{BiOBr}$-circle sample exhibits an inhomogeneous atomic arrangement across the nanosheet in figure 2(e). The areas close to the edge exhibit relaxation in their atomic structures, although the central area retains a similar atomic arrangement to that in the square nanosheet. The FFT pattern, shown in figure 2(g), exhibits a group of symmetric spots (pattern B, labelled in green) in addition to the intrinsic spots (pattern A, labelled in yellow). These spots of pattern B with a shorter nearest-neighbor spot distance are attributed to the area close to the edge (Area B) in figure 2(f). Their presence reflects the considerable lattice relaxation exists in the areas close to the edges, as illustrated in figures S6 and S7 in Supporting Information. These relaxed areas induce a significantly inhomogeneous distribution of inner strain in the BiOBr-circle nanosheets. It should be noted that a sharp interface can be observed between Area A and Area B in the BiOBr-circle nanosheets, as indicated by the dashed lines in figure 2(e) and (f). This interface can be regarded as a lateral 
pseudoheterointerface, because both areas have identical chemical component composition but distinctly different inner strains (see more TEM images in figures S8 and S9 in Supporting Information). In order to revealing the detailed local strain distribution, we used the geometric phase analysis (GPA) method to study both square and circle nanosheets [35,36]. Figure 2(d) and (h) show GPA strain mapping results for the BiOBr-square and the BiOBr-circle nanosheets, respectively. As shown in figure 2(d), the distribution of local strain is uniform despite the subtle fluctuation in the $\mathrm{BiOBr}$-square nanosheets. Whereas, figure 2(h) shows that the inner strains of Area A and Area B in the BiOBr-circle nanosheet are different, with a clear interface between these two areas (see more results in figure S10 in Supporting Information). Our high resolution TEM (HRTEM) images and GPA suggest that the strain distribution in the $\mathrm{BiOBr}$ nanosheets can be manipulated through controlling the concentration of hydroxide ( $\mathrm{pH}$ value) in the hydrothermal reactions. Lateral pseudoheterostructures based on inhomogeneous strain distribution can be obtained in a single $\mathrm{BiOBr}$ nanosheet.
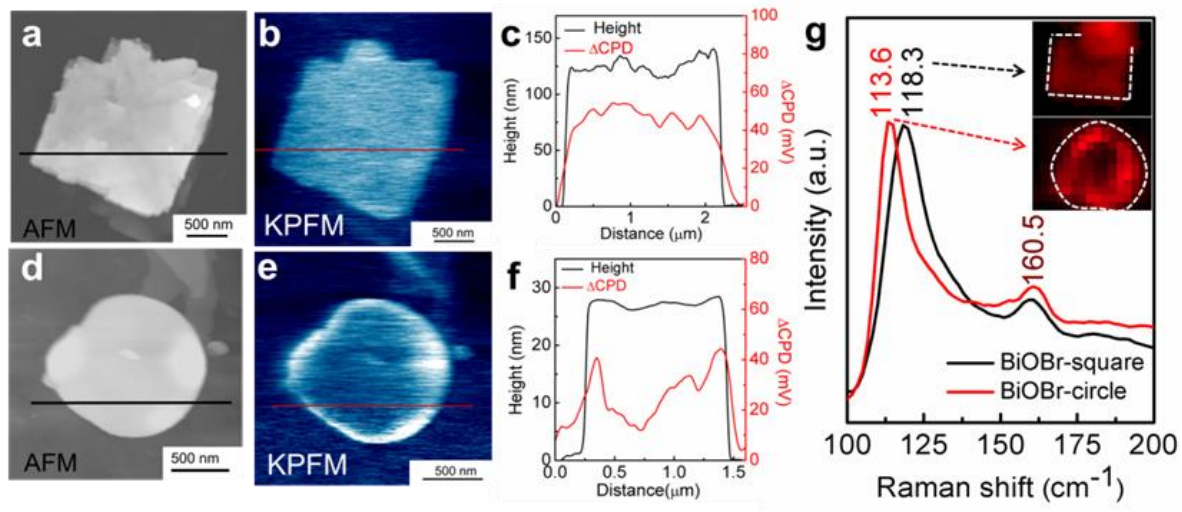

Figure 3. (a) AFM image of $\mathrm{BiOBr}$-square. (b) KPFM image of $\mathrm{BiOBr}$-square. (c) Corresponding line profiles of the lines in a and $b$, with the height as a black line and the 
work function as a red line. (d) AFM image of BiOBr-circle. (e) KPFM image of BiOBr-circle. (f) The corresponding line profiles of the lines in $\mathrm{d}$ and e, with the height as a black line and work function as a red line. (g) Raman spectra of $\mathrm{BiOBr}$ nanosheets, with $\mathrm{BiOBr}$-square shown by the black line and $\mathrm{BiOBr}$-circle by the red line. Insets are the Raman mappings at $118.3 \mathrm{~cm}^{-1}$ of $\mathrm{BiOBr}$-square (top) and $113.6 \mathrm{~cm}^{-1}$ of $\mathrm{BiOBr}$-circle (bottom).

Generally, the local electronic structures in 2D materials can be significantly affected by local strain $[37,38]$. The surface morphologies and local work functions of BiOBr nanosheets were determined by Kelvin probe force microscope (KPFM). As shown in figure 3(a) and (d), both square and circle $\mathrm{BiOBr}$ nanosheets present a flat surface. The morphologies are consistent with TEM results. Figure 3(b) and (e) show the local work function mapping images acquired simultaneously with atomic force microscope (AFM) measurements on square and circle $\mathrm{BiOBr}$ nanosheets, respectively. By measuring the contact potential difference (CPD) between the conductive tip with a constant work function and the surface, KPFM can precisely determine the work function of materials with high resolution [39]. The $\mathrm{BiOBr}$-square nanosheet shows a homogeneous work function distribution across its entire surface, as demonstrated by the line profiles in figure 3(c). In contrast, the work function on the $\mathrm{BiOBr}$-circle nanosheet exhibits an apparent variation between the area near the edge and the central area, as shown in figure 3(e) and the line profile in figure 3(f) (see also the sample with intermediate shape in figure S11 in Supporting Information). We conjecture that the variation in the work function might be attributable to distinct local electronic structures induced by the inhomogeneous distribution of local strains beside the lateral 
pseudoheterointerface in $\mathrm{BiOBr}$-circle nanosheets. In order to verify this hypothesis, confocal Raman spectroscopy was employed (incident laser wavelength $\lambda=532 \mathrm{~nm}$ ) to identify the correlation between the work function and the local strain in $\mathrm{BiOBr}$ nanosheets, as shown in figure 3(g). Two strong phonon modes can be observed in the Raman spectra of the $\mathrm{BiOBr}$ samples. The Raman peaks of BiOBr-square are located at $118.3 \mathrm{~cm}^{-1}$ and $160.5 \mathrm{~cm}^{-1}$, while the Raman peaks of BiOBr-circle are located at $113.6 \mathrm{~cm}^{-1}$ and $160.5 \mathrm{~cm}^{-1}$. According to previous reports, the peak around $113.6 \mathrm{~cm}^{-1}$ can be assigned to the $\mathrm{A}_{1 \mathrm{~g}}$ mode (internal $\mathrm{Bi}-\mathrm{Br}$ stretching), and the peak at $160.5 \mathrm{~cm}^{-1}$ can be assigned to the $\mathrm{E}_{\mathrm{g}}$ mode (internal $\mathrm{Bi}-\mathrm{Br}$ stretching) [40]. Although the $\mathrm{E}_{\mathrm{g}}$ mode remains at $160.5 \mathrm{~cm}^{-1}$ for both samples, the $\mathrm{A}_{1 \mathrm{~g}}$ peak of $\mathrm{BiOBr}$-square possesses a blue shift of $\sim 5 \mathrm{~cm}^{-1}$ from $113.6 \mathrm{~cm}^{-1}$ to $118.3 \mathrm{~cm}^{-1}$, which suggests a large inner compressive strain. Raman mapping of the $\mathrm{A}_{\lg }$ peak on both square and circle nanosheets were carried out to provide a straightforward way to identify the strain distributions. As shown in the insets in figure $3 \mathrm{~g}$, the intensity of the peak at $118.3 \mathrm{~cm}^{-1}$ for BiOBr-square was homogenously recorded across the whole nanosheet, indicating the homogeneous distribution of strain. In contrast, for BiOBr-circle, the intensity of the peak at $113.6 \mathrm{~cm}^{-1}$ is much stronger near the edge area than in the central area. This observation is supported by the fact that the relaxation of strain sprang from the edge area to the inner central area in $\mathrm{BiOBr}$-circle nanosheets. It should be notice that during the Raman mapping, the resolution of is limited by the size of the laser spot, which is around $361 \mathrm{~nm}$ in this work. The spatial variation in the $A_{1 \mathrm{~g}}$ peak is on a smaller scale than the resolution of the instrument, and so there is definitely some averaging between each analysis spot, which will border the Raman activate area in the measurement. These results demonstrate that the variation of the 
local electronic structure can be directly correlated with its inhomogeneous strain distribution beside the lateral pseudoheterointerface in the $\mathrm{BiOBr}$-circle nanosheet.
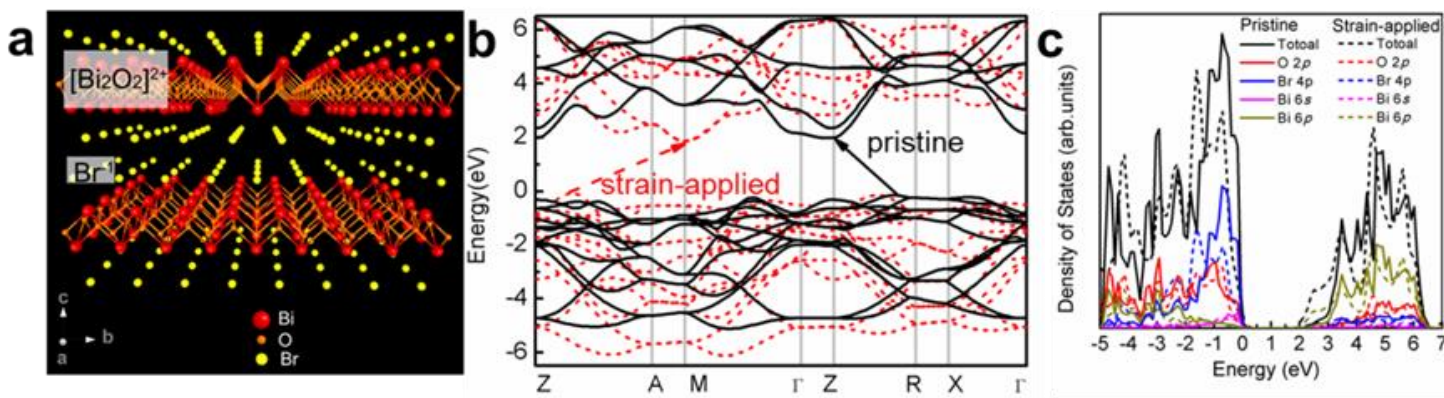

Figure 4. (a) Crystallographic structure of $\mathrm{BiOBr}$. (b) Calculated band structure of $\mathrm{BiOBr}$, with the pristine $\mathrm{BiOBr}$ shown by the black solid line and the $\mathrm{BiOBr}$ with compressive strain shown by the red dashed line. (c) Calculated DOS of $\mathrm{BiOBr}$, with the pristine $\mathrm{BiOBr}$ shown by the solid line and $\mathrm{BiOBr}$ with compressive strain shown by the dashed line.

We carried out density functional theory (DFT) calculations to simulate the strain dependence of the electronic structure in $\mathrm{BiOBr}$. As illustrated in figure 4(a), the layered crystal structure of $\mathrm{BiOBr}$ consists of $\left[\mathrm{Bi}_{2} \mathrm{O}_{2}\right]^{2+}$ and $\mathrm{Br}^{-}$slabs along the $[001]$ direction with weak vdW interactions joining two closely adjacent $\mathrm{Br}^{-}$layers. Figure 4(b) and (c) show DFT calculation results for the pristine and strain-applied BiOBr. When an in-plane strain (for example, the compressive strain here) is applied, the band structure and density of states (DOS) of $\mathrm{BiOBr}$ are significantly modulated. The band gap is decreased by $0.2 \mathrm{eV}$ in $\mathrm{BiOBr}$ under compressive strain (2.12 eV for pristine $\mathrm{BiOBr})$. The conduction band minimum (CBM) shifts from the $\mathrm{Z}$ point to the $\mathrm{M}$ point in the irreducible Brillouin zone, and the 
valence band maximum (VBM) moves from its position between the $\mathrm{R}$ and $\mathrm{Z}$ points to a position between the $\mathrm{Z}$ and $\mathrm{A}$ points. The most attractive feature in the electronic structure is that the band structure around the $\mathrm{CBM}$ is more dispersive in strained $\mathrm{BiOBr}$ than in pristine $\mathrm{BiOBr}$. This suggests that the excited electrons in strained $\mathrm{BiOBr}$ have a small effective mass and high mobility. As a result, the total DOS of BiOBr can also be tuned by strain, as shown in figure 4(c), in which the partial DOS contributed by the O $2 p$ orbitals is significantly changed. The change in the electronic structure can be ascribed to the symmetry transition in the $\mathrm{BiOBr}$ crystal under strain, which is a common phenomenon in many other semiconductors [41]. The DFT calculations indicate that the distinct electronic properties flanking the pseudoheterointerface in the $\mathrm{BiOBr}$-circle nanosheets are indeed controlled by the local strain distribution, which supports our experimental findings.

To verify the ability of separating photoexcited charge carriers in $\mathrm{BiOBr}$ nanosheets, photodetectors were fabricated on $\mathrm{BiOBr}$-square (figure 5(c)) and $\mathrm{BiOBr}$-circle (figure 5(d)) nanosheets, respectively, via electron-beam lithography. We measured the photocurrent and photoresponse of both $\mathrm{BiOBr}$ nanosheets. As shown in figure 5(a), under visible light irradiation $\left(450 \mathrm{~nm}, 50 \mathrm{~mW} \cdot \mathrm{cm}^{-2}\right)$, photocurrent was generated in both BiOBr-square and $\mathrm{BiOBr}$-circle nanosheets. The photocurrent generated in the BiOBr-circle nanosheet is about $2 \mathrm{nA}$, which is nearly one order of magnitude larger than that of the $\mathrm{BiOBr}$-square nanosheet under the same experimental conditions. (see the calculated responsivity and external quantum efficiency in Supporting Information) 

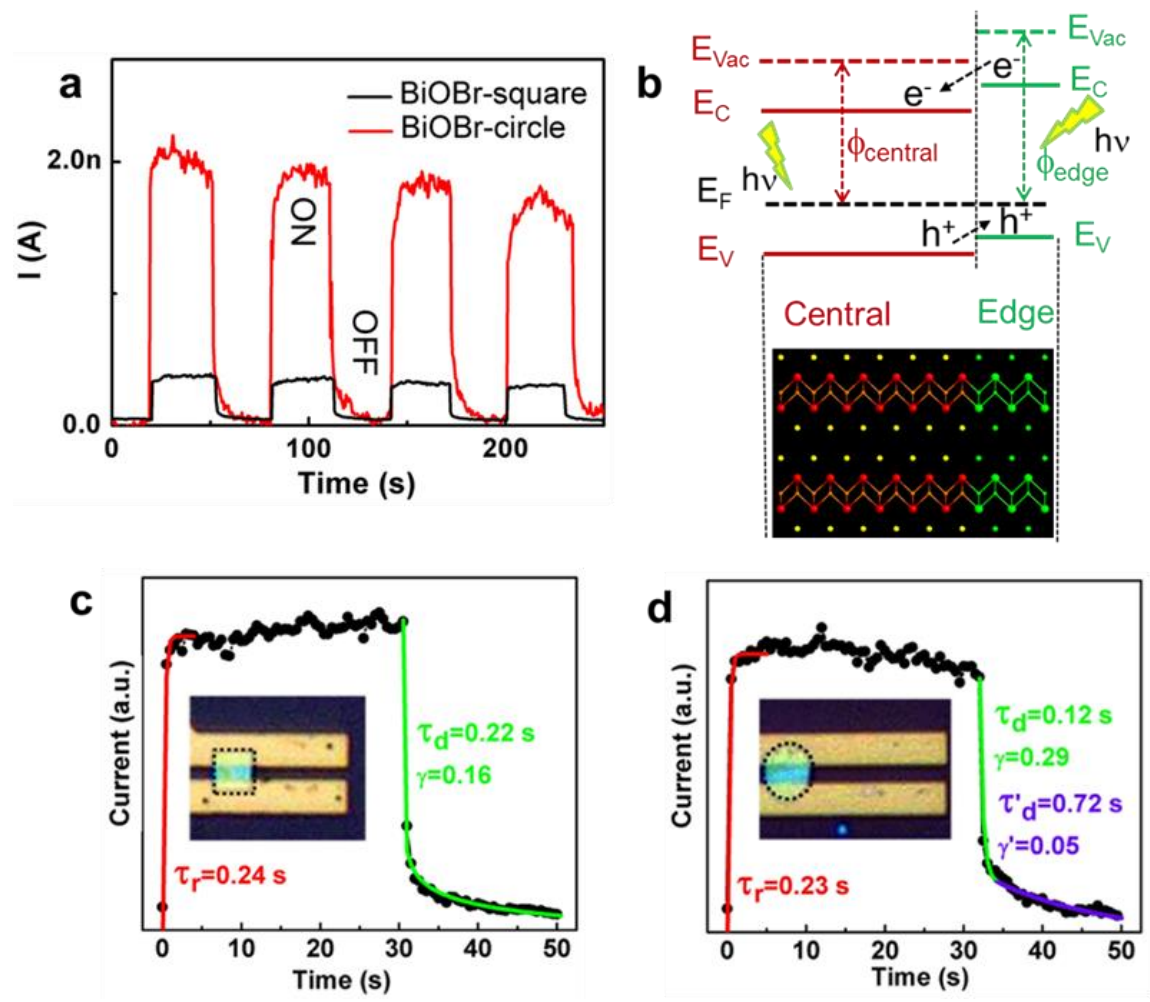

Figure 5. (a) Photocurrent of individual $\mathrm{BiOBr}$ nanosheets. (b) Schematic diagram of the band alignment at the pseudoheterointerface in $\mathrm{BiOBr}$-circle, which promotes the separation of photoexcited carriers. (c) Acquiring rise and decay time of $\mathrm{BiOBr}$-square by fitting the on/off curve, inset is an image of the nanodevice. (d) Acquiring rise and decay time of BiOBr-circle by fitting the on/off curve, inset is the image of the device.

In addition to the contrasting photocurrents in these two $\mathrm{BiOBr}$ nanosheets, a difference in the photoresponse between $\mathrm{BiOBr}$-square and $\mathrm{BiOBr}$-circle nanosheets can be observed in figure 5(a). Generally, the dynamic response of the rise (equation (1)) and decay (equation (2) and (3)) of photocurrent in $\mathrm{BiOBr}$ nanosheets can be fitted by the following stretched exponential functions: 


$$
\begin{gathered}
I=I_{0}-I_{0} \mathrm{e}^{\left(\frac{-\mathrm{t}}{\tau_{\gamma}}\right)^{\gamma}} \\
I=I_{0} \mathrm{e}^{\left(\frac{-\mathrm{t}}{\tau_{\mathrm{d}}}\right)^{\gamma}} \\
I=\mathrm{A} I_{0} \mathrm{e}^{\left(\frac{-\mathrm{t}}{\tau_{\mathrm{d}}}\right)^{\gamma}}+\mathrm{B} I_{0} e^{\left(\frac{-t}{\tau^{\prime} d}\right)^{\gamma \prime}}
\end{gathered}
$$

where $\tau_{\mathrm{r}}$ and $\tau_{\mathrm{d}}$ are the relaxation time constants of the rise and decay, respectively. The parameter $\gamma$ falls into the range of 0 to 1 . As $\gamma$ approaches 1 , the function approaches classic single-exponential behavior without stretching. We have fitted the photoresponse curves of the $\mathrm{BiOBr}$ nanosheets to reveal the dynamic responses under visible light irradiation. As shown in figure 5(c), the $\tau_{\mathrm{r}}$ for $\mathrm{BiOBr}$-square is calculated to be $0.24 \mathrm{~s}$ (equation (1)) The value of $\gamma$ is calculated as 1, which suggests that the generation of photocurrent in BiOBr-square under light irradiation is dominated by the separation of photoexcited electron-hole pairs. The decay of photocurrent for BiOBr-square can be described by a stretched exponential function (equation (2)), where the value of $\tau_{\mathrm{d}}$ is $0.22 \mathrm{~s}$ and the value of $\gamma$ is 0.16 , suggesting that the recombination of photocurrent involves multiple energy processes, which may origin from the strain-induced distortion of the crystal lattice. For BiOBr-circle, as shown in figure $5(\mathrm{~d})$, the rise of photocurrent could also be described using single exponential functions with $\tau_{\mathrm{r}}$ of $0.23 \mathrm{~s}$, which is almost the same as for $\mathrm{BiOBr}$-square. To describe the decay of photocurrent in BiOBr-circle, however, at least two components are necessary to fit the persistent photocurrent decay, where two exponential functions (equation (3)) are used to separately analyze two different photorelaxation processes. The $\tau_{\mathrm{d}}$ of the fast process was determined to be $0.12 \mathrm{~s}$ with $\gamma$ as 0.29 , and the $\tau^{\prime}{ }_{\mathrm{d}}$ of the slow process was calculated to be $0.72 \mathrm{~s}$ with $\gamma$ as 0.05 . During the photocurrent decay, it is well known that 
photoexcited electron-hole recombination dominates the fast decay process in the photocurrent. Hence, the photocurrent decreases very rapidly in the initial stage. The slow process is caused by a mechanism in which the photoexcited carriers are trapped and spatially separated by local potential fluctuations, which can suppress the electron-hole recombination. For our samples, because both the $\mathrm{BiOBr}$-square and $\mathrm{BiOBr}$-circle nanosheets exhibit defect-free characteristics, supported by the homogeneous element distribution across the whole nanosheets (EDS element mapping in figure S4 in Supporting Information). Therefore, it is believed that the slow relaxation process in $\mathrm{BiOBr}$-circle, which reflects the longer lifetime of photoexcited charge carriers, is governed by the strain-induced pseudoheterointerface.

We attribute the significant enhancement of the photocurrent in the $\mathrm{BiOBr}$-circle nanosheets to improved separation efficiency of photoexcited charge carriers, which is illustrated in figure 5(b). In the BiOBr-circle nanosheets, the inhomogeneous work function $(\phi)$ distribution beside the lateral pseudoheterointerface, as shown in figure 3 , induces band bending across the interface [42]. Therefore, under light irradiation, photoexcited electrons and holes will be spatially driven by this electric field and separate to two different regions in the nanosheet, which will prolong their lifetime and consequently enhance the light harvesting efficiency in the $\mathrm{BiOBr}$-circle nanosheets. In contrast, there are not any pseudoheterointerface in BiOBr-square nanosheets. Therefore, we do not expect the same scenario occurring in square nanosheets. 


\section{Conclusion}

To summarize, the lateral pseudoheterostructure on individual $\mathrm{BiOBr}$ nanosheets was fabricated by strain engineering. The local strain distribution has been revealed by TEM and Raman mapping. The dependence of local strain on electronic structures has been revealed by DFT calculations. An inhomogeneous work function distribution is observed across the lateral pseudoheterostructure, which favors the separation of photoexcited charge carriers under visible light irradiation. Enhanced photocurrent (by one order of magnitude) has been achieved in BiOBr-pseudoheterostructure-based photodetectors, which is attributed to the appropriate band alignment across the pseudoheterointerface. The growth of such lateral pseudoheterostructures, thus, offers a promising way to build systems that show the functionalities of 2D lateral heterostructures but overcome the problems of lattice mismatch.

\section{Method}

To synthesize $\mathrm{BiOBr}$ nanosheets, $5 \mathrm{mmol}$ Bismuth nitrate pentahydrate and $5 \mathrm{mmol}$ cetyl trimethylammonium bromide $(\mathrm{CTAB})$ were, respectively, added into $100 \mathrm{~mL}$ distilled water at room temperature. Sodium hydroxide solution (1 M) was added to the mixed solution to adjust the $\mathrm{pH}$ value. The mixed solution was then stirred for $1 \mathrm{~h}$, and poured into a $100 \mathrm{~mL}$ Teflon-lined stainless autoclave up to $80 \%$ of the total volume. In the hydrothermal reaction, the sealed autoclave was heated at $170{ }^{\circ} \mathrm{C}$ for $17 \mathrm{~h}$, and then cooled in air. The resulting precipitates were collected, washed with ethanol and deionized water several times, and dried at $80{ }^{\circ} \mathrm{C}$ for $10 \mathrm{~h}$. Bismuth nitrate pentahydrate, Sodium hydroxide, and CTAB were purchased from Sinopharm Chemical Reagent Co., Ltd. (SCRC). All reagents used in this work were of analytical grade and were used as received without any further purification. 
The morphologies and microstructures of the as-prepared samples were characterized by SEM (JEOL JSM-7500FA) and HAADF images were obtained by STEM (JEOL JEM-ARM200F). The GPA strain maps were carried out with STEM-Cell software using the HAADF images. In the analysis process, the displacement of lattice parameters (u) are determined by calculating and analyzing the Fourier transform of the selected image. The strain is then obtained from the derivative of the displacement in the picked direction, and visualized by inverse Fourier transform. A commercial AFM (Asylum Research MFP-3D) was used to measure the morphology and surface potential of the $\mathrm{BiOBr}$ nanosheets by SKPM. A Pt/Ir coated n-silicon probe with resonance frequency of $45-115 \mathrm{kHz}$ and force constant of $0.5-0.95 \mathrm{M} / \mathrm{m}$ was used in the AFM measurements. BiOBr powders were distributed on gold $(50 \mathrm{~nm})$ coated silicon substrates. Before characterization of the surface potential of $\mathrm{BiOBr}$ samples, the surface potential of the Pt/Ir tip was calibrated on a standard Si wafer coated with Au film. Raman mapping investigations were carried out with a confocal Raman spectrometer (Horiba Xplora) using a 532-nm wavelength laser as the excitation source.

The first-principles calculations were performed using the Vienna Ab initio Simulation Package (VASP) code. The generalised gradient approximation (GGA) was applied to treat the exchange correlation energy with the Perdew-Burke-Ernzerhof (PBE) functional. The projector-augmented wave (PAW) method was employed to describe the electron-ion interactions. A k-point sampling of a $9 \times 9 \times 6$ grid was generated with original Gamma-centred meshes. The cut-off energy for the plane wave basis was $550 \mathrm{eV}$. The biaxial strain 
simulations were realized by fixing the $\mathrm{x}$ and $\mathrm{y}$ axes and optimizing the $\mathrm{z}$ axis. Equilibrium geometries were obtained by the minimum energy principle.

A pair of electrical contacts (Ti/Au, $5 / 50 \mathrm{~nm}$ thick) was fabricated on the a $\mathrm{SiO}_{2} / \mathrm{Si}$ surface, by standard electron beam lithography, using a poly (methyl methacrylate) resist and the lift-off method. BiOBr nanosheets were dispersed in ethanol and then dropped on the as-prepared surface followed by air-drying at $60{ }^{\circ} \mathrm{C}$. Flakes suitable for electrical characterization were identified by an optical microscope. Photocurrent measurements were performed in vacuum at room temperature, using a probe station connected to a semiconductor parameter analyser (Agilent B1500). The drain bias of $10 \mathrm{~V}$ and a gate bias of $80 \mathrm{~V}$ have been applied. The wavelength of the light source was $450 \mathrm{~nm}$. The power of the light was measured to be $50 \mathrm{~mW} \cdot \mathrm{cm}^{-2}$.

\section{Supplementary material for this article is available online}

\section{Acknowledgements}

This work is supported by the Australian Research Council (ARC) through Discovery Projects (DP140102581, DP160102627 and DP170101467), the National Natural Science Foundation of China (Nos. 51472016, 51272015), and Fundamental Research Funds for the Central Universities (YWF-16-JCTD-B-03). This work was performed in part at the South Australian node of the Australian National Fabrication Facility (ANFF), a company established under the National Collaborative Research Infrastructure Strategy to provide nano and micro-fabrication facilities for Australia's researchers. Australian Microscopy and 
Microanalysis Research Facility (AMMRF) supported the Raman facility along with ANFF. The authors acknowledge use of facilities within the UOW Electron Microscopy Centre. The authors thank Dr. T. Silver for her valuable comments on this work.

\section{Author contributions}

W.H. and Y. D. conceived the project and supervised the research; H.F., W.H. and Y.D. designed the experiments; H.F. J.Z., L.W. and L.R. conducted synthesis; H.F. and A.D.S. conducted Raman characterizations and analysis; Z.X. and W.H. conducted computational modelling and simulations; D.M. conducted TEM characterizations; H.F., Y.D., T.Z. and M.H. carried out AFM and KPFM characterizations; X.X., Z.S. and S. L. designed and manufactured devices; H.F., S.X.D., Y.D. and W.H. wrote the manuscript. All authors contributed to data analysis, discussions and manuscript preparation.

\section{References}

[1] Hwang H Y, Iwasa Y, Kawasaki M, Keimer B, Nagaosa N and Tokura Y 2012 Emergent phenomena at oxide interfaces Nat. Mater. 11 103-113

[2] Alferov Z I 2001 Nobel lecture: the double heterostructure concept and its applications in physics, electronics, and technology Rev. Mod. Phys. 73 767-782

[3] Keimer B, Kivelson S A, Norman M R, Uchida S and Zaanen J 2015 From quantummatter to high-temperature superconductivity in copper oxides Nature 518 179-186 
[4] Nakayama K, Eto K, Tanaka Y, Sato T, Souma S, Takahashi T, Segawa K and Ando Y 2012 Manipulation of topological states and the bulk band gap using natural heterostructures of a topological insulator Phys. Rev. Lett. 109236804

[5] Ohtomo A and Hwang H Y 2004 A high-mobility electron gas at the $\mathrm{LaAlO}_{3} / \mathrm{SrTiO}_{3}$ heterointerface Nature $\mathbf{4 2 7}$ 423-426

[6] Li L, Richter C, Mannhart J and Ashoori R C 2011 Coexistence of magnetic order and two-dimensional superconductivity at $\mathrm{LaAlO}_{3} / \mathrm{SrTiO}_{3}$ interfaces Nat. Phys. 7 762-766

[7] Dean C R et al 2010 Boron nitride substrates for high-quality graphene electronics Nat. Nanotechnol. 5 722-726

[8] Wang M et al 2013 Platform for large-scale graphene electronics-CVD growth of single-layer graphene on CVD-grown hexagonal boron nitride Adv. Mater. 25 2746-2752

[9] Geim A K and Grigorieva I V 2013 Van der Waals heterostructures Nature 499 419-425

[10] Haigh S J, Gholinia A, Jalil R, Romani S, Britnell L, Elias D C, Novoselov K S, Ponomarenko L A, Geim A K and Gorbachev R 2012 Cross-sectional imaging of individual layers and buried interfaces of graphene-based heterostructures and superlattices Nat. Mater. $11764-767$

[11] Massicotte M, Schmidt P, Vialla F, Schädler K G, Reserbat-Plantey A, Watanabe K, Taniguchi T, Tielrooij K J and Koppens F H L 2016 Picosecond photoresponse in van der Waals heterostructures Nat. Nanotechnol. 11 42-46 
[12] Liu Y, Weiss N O, Duan X, Cheng H-C, Huang Y and Duan X 2016 Van der Waals heterostructures and devices Nat. Rev. Mater. 116042

[13] Liu L, Park J, Siegel D A, McCarty K F, Clark K W, Deng W, Basile L, Idrobo J C, Li A-P and Gu G 2014 Heteroepitaxial growth of two-dimensional hexagonal boron nitride templated by graphene edges Science 343 163-167

[14] Levendorf M P, Kim C-J, Brown L, Huang P Y, Havener R W, Muller D A and Park J 2012 Graphene and boron nitride lateral heterostructures for atomically thin circuitry Nature $488627-632$

[15] Duan X et al 2014 Lateral epitaxial growth of two-dimensional layered semiconductor heterojunctions Nat. Nanotechnol. 9 1024-1030

[16] Huang C, Wu S, Sanchez A M, Peters J J P, Beanland R, Ross J S, Rivera P, Yao W, Cobden D H and Xu X 2014 Lateral heterojunctions within monolayer $\mathrm{MoSe}_{2}-\mathrm{WSe}_{2}$ semiconductors Nat. Mater. 13 1096-1101

[17] Zhang X-Q, Lin C-H, Tseng Y-W, Huang K-H and Lee Y-H 2015 Synthesis of lateral heterostructures of semiconducting atomic layers Nano Lett. 15 410-415

[18] Son Y, Li M-Y, Cheng C-C, Wei K-H, Liu P, Wang Q H, Li L-J and Strano M S 2016 Observation of switchable photoresponse of a monolayer $\mathrm{WSe}_{2}-\mathrm{MoS}_{2}$ lateral heterostructure via photocurrent spectral atomic force microscopic imaging Nano Lett. 16 3571-3577 
[19] Novoselov K S, Mishchenko A, Carvalho A and Neto A H C 2016 2D materials and Van der Waals heterostructures Science 353461

[20] Wang H, Liu F, Fu W, Fang Z, Zhou W and Liu Z 2014 Two-dimensional heterostructures: fabrication, characterization, and application Nanoscale 6 12250-12272

[21] Nam D, Sukhdeo D S, Kang J-H, Petykiewicz J, Lee J H, Jung W S, Vučković J, Brongersma M L and Saraswat K C 2013 Strain-induced pseudoheterostructure nanowires confining carriers at room temperature with nanoscale-tunable band profiles Nano Lett. $\mathbf{1 3}$ $3118-3123$

[22] Feng J, Qian X, Huang C-W and Li J 2012 Strain-engineered artificial atom as a broad-spectrum solar energy funnel Nat. Photonics 6 866-872

[23] An H, Du Y, Wang T, Wang C, Hao W and Zhang J 2008 Photocatalytic properties of $\mathrm{BiOX}(\mathrm{X}=\mathrm{Cl}, \mathrm{Br}$, and I) Rare Metals 27 243-250

[24] Zhang K-L, Liu C-M, Huang F-Q, Zheng C and Wang W-D 2006 Study of the electronic structure and photocatalytic activity of the BiOCl photocatalyst Appl. Catal. B. 68 125-129

[25] Zou W, Hao W-C, Xin X and Wang T-M 2009 Visible-light photocatalytic degradation of RhB by $\mathrm{Bi}_{2} \mathrm{O}_{3}$ polymorphs Chinese J. Inorg. Chem. 25 1971-1976

[26] Hao W, Gao Y, Jing X, Zou W, Chen Y and Wang T 2014 Visible light photocatalytic properties of metastable $\gamma-\mathrm{Bi}_{2} \mathrm{O}_{3}$ with different morphologies J. Mater. Sci. Technol. 30 $192-196$ 
[27] Cheng H, Huang B and Dai Y 2014 Engineering BiOX (X = Cl, Br, I) nanostructures for highly efficient photocatalytic applications Nanoscale 6 2009-2026

[28] Li H, Shang J, Ai Z and Zhang L 2015 Efficient visible light nitrogen fixation with BiOBr nanosheets of oxygen vacancies on the exposed \{001\} facets J. Am. Chem. Soc. 137 6393-6399

[29] Xu Z, Han L, Lou B, Zhang X and Dong S 2014 High-performance BiOBr ultraviolet photodetector fabricated by a green and facile interfacial self-assembly strategy Nanoscale $\mathbf{6}$ $145-150$

[30] Li H, Hu T, Liu J, Song S, Du N, Zhang R and Hou W 2016 Thickness-dependent photocatalytic activity of bismuth oxybromide nanosheets with highly exposed (010) facets Appl. Catal. B 182 431-438

[31] Andrés J, Gracia L, Gouveia A F, Ferrer M M and Longo E 2015 Effects of surface stability on the morphological transformation of metals and metal oxides as investigated by first-principles calculations Nanotechnology 26405703

[32] Zhang H, Liu L and Zhou Z 2012 First-principles studies on facet-dependent photocatalytic properties of bismuth oxyhalides (BiOXs) RSC Adv. 2 9224-9229

[33] Feng $\mathrm{H}$ et al 2015 Modulation of photocatalytic properties by strain in $2 \mathrm{D} \mathrm{BiOBr}$ nanosheets ACS Appl. Mater. Interfaces 7 27592-27596

[34] Dandekar P, Kuvadia Z B and Doherty M F 2013 Engineering crystal morphology Annu. Rev. Mater. Res. 43 359-386 
[35] Grillo V and Rotunno E 2013 STEM_CELL: a software tool for electron microscopy: part I simulations Ultramicroscopy 125 97-111

[36] Grillo V and Rossi F 2013 STEM_CELL: a software tool for electron microscopy. part 2 analysis of crystalline materials Ultramicroscopy 125 112-129

[37] Guinea F, Katsnelson M I and Geim A K 2010 Energy gaps and a zero-field quantum hall effect in graphene by strain engineering Nat. Phys. 6 30-33

[38] Xu Z, Hao W, Zhang Q, Fu Z, Feng H, Du Y and Dou S 2016 Indirect-direct band transformation of few-layer BiOCl under biaxial strain. J. Phys. Chem. C 120 8589-8594

[39] Palermo V, Palma M and Samorì P 2006 Electronic characterization of organic thin films by kelvin probe force microscopy Adv. Mater. 18 145-164

[40] Davies J E D 1973 Solid state vibrational spectroscopy - III [1] the infrared and Raman spectra of the bismuth (III) oxide halides J. Inorg, Nucl. Chem. 35 1531-1534

[41] Hong K-H, Kim J, Lee S-H and Shin J K 2008 Strain-driven electronic band structure modulation of Si nanowires Nano Lett. 8 1335-1340

[42] Kahn A 2016 Fermi level, work function and vacuum level Mater. Horiz. 3 7-10 